\title{
A Novel Visual Saliency Guided High Resolution Remote Sensing Image Change Detection Algorithm
}

\author{
Qigejian Wang ${ }^{1, \text { a }}$, Xinyu Zhang ${ }^{2}$ \\ ${ }^{1}$ Huazhong University of Science and Technology, Wuhan, China; \\ ${ }^{2}$ Graduate School of Art and Science (Statistics), Columbia University, New York, USA. \\ awangqigejian15@sina.com
}

Keywords: Visual Saliency, Remote Sensing, Change Detection, High Resolution Image.

\begin{abstract}
This paper presents a novel visual saliency guided high resolution remote sensing image change detection (CD) algorithm. This approach is based on closed-loop process made up of the three procedures. (1) Generating the difference image as the foundation for further processing; (2) Use the modified saliency extraction algorithm to select the regions with higher attractiveness; (3) Adopt the revised fuzzy clustering to finalize the real changed regions (RCR). Our algorithm could fuse the traditional change analysis patterns with the saliency prior which could enhance the overall efficiency. Experiments carried out on the different sets of high resolution multi-temporal images acquired by the satellite. The experimental result shows that our method could achieve better performance.
\end{abstract}

\section{Introduction}

The accumulation of remote sensing data and the establishment of space database fully recorded the data of different spatial scales of the earth's surface. From the information extraction and the change detection in remote sensing data is the key of the remote sensing information science research subject. Remote sensing image change detection tasks take the feature as the research object, including natural features and man-made features. The change of terrain features, such as the disappearance of terrain and the appearance changes in the structure will cause the change of the remote sensing images. In addition, the sun angle, atmospheric conditions, the sensor precision, factors such as the soil moisture condition and phenological period features can cause changes in remote sensing images [1].

In recent years, with the help of the international scholars, change detection method has developed to a great extent. According to different classification standards, we can divide these methods into the listed categories. (1) Image regression methods. Regression analysis by the least squares method, and then reuse the regression equation to calculate the predicted value minus the images of real value to obtain the return of the two images difference image while the image can be used to reflect the land cover change information. (2) Spectral change vector analysis. Spectral change vector analysis based method is based on the differences by the radiation between the phase images during the same time, analyzes the differences of each band to determine strength and direction of change. (3) Vegetation index difference method. Using near infrared wave band optical sensor and the infrared wavelengths significant response difference of vegetation by ratio of outstanding vegetation information, and then through the threshold to extract vegetation information. (4) Principal component analysis. Principal component analysis is able to separate the advantages of the information, reduce the related, so as to highlight the different features. Its disadvantages is that it is based on pure statistical relation, thus it produces the amount of physical meaning is not clear, and can only reflect the change of distribution and the size which causes difficulty on expressing the type of change [2].

To optimize the traditional method, in this paper, we propose the novel visual saliency guided high resolution remote sensing image change detection algorithm. Compared with the classical approaches that mainly focus on the overall change pattern discovery and analysis, saliency guided algorithm will emphasis more on the significant change area which could enhance the detection efficiency and is closer to the actual applications. The rest of the paper is organized as the follows. We firstly review 
the state-of-the-art visual saliency extraction algorithm to select the proper one as the pre-processing procedure of the change detection task. Later, we combine the fuzzy clustering and local information fusion technique to form the primary component of our change detection algorithm, then, to test the feasibility and efficiency, we simulation the proposed algorithm numerically and visually. Finally, we summarize the study and set up the orientation for our further research.

\section{Our Proposed Algorithm}

Visual Saliency Extraction Methodology. In the field of computer vision, saliency test is a direction in the study of visual attention mechanism. Significant testing embodied in a given image, determine the image of visual attention every area of ability, and through the grey quantitative characterization of the significance of the image. Visual significance measure is the most commonly used method to realize guidance of the visual attention, namely various visual feature maps transform to significant figure. In the classical model, a significant degree is only on the basis of local information of object area and its neighborhood, without considering the global information of the image. Therefore, we combine the global and local information to modify the saliency extraction steps [3].

The significance of a pixel in the image depends on its own characteristics with the surrounding environment difference, the greater the differences in the features of the surrounding environment, the more significant it will be. As a result, we could consider use each pixel in the image characteristics of adjacent pixels difference to measure the pixels in a local neighborhood of the visual significance, namely local visual significant measure. In the formula 1 2 we describe the saliency finding steps.

$$
\begin{aligned}
& H(\text { Image })=H(\text { Innovation })+H(\text { Prior }) \\
& H(\text { Innovation })=H(\text { Image })-H(\text { Prior })
\end{aligned}
$$

The $H$ (Image) represents the information contained in the single image, $H$ (Prior) denotes prior knowledge, $H$ (Innovation) represents the new information. Under this guidance, we could capture the saliency map according to the spectral residual error and phase spectrum information as follows.

$$
\text { Saliency }(x)=G(x) *\left|F^{-1}[\exp (R(f)+P(f))]\right|^{2}
$$

Local significance measure considering the pixel to be relative to local area around its significance, only local significance measure will appear "significant reversal" or have a local effect. To eliminate the influence and the drawbacks, we consider pixels characteristic difference of relative to the whole image with globally significant measure. Through calculating each pixel and the characteristics of the other pixels in the image difference, we regard the difference between the mean as the pixels of global significant degree to form the enhanced significant figure. To further strengthen the competition between space positions and widened significantly with prominent position, the significant difference between the processes are treated for multiple iterations as the equation 4.

$$
\left\{\begin{array}{c}
Q S_{i j}^{0}(x, y)=F_{i j}(x, y) \\
Q S_{i j}^{l+1}(x, y)=\frac{\sum\left|Q S_{i j}^{l}(x, y)-v\right| H_{Q S_{i j}^{l}}(v)}{(\text { row } \times \text { col }-1)}
\end{array}\right.
$$

$F_{i j}$ represents the characteristic pattern of class $j, Q S_{i j}^{l}(x, y)$ is the globally significant figure after $l$ th iteration, row and col represent the corresponding rows and columns, respectively. Based on this feature, we combine the latest weight calculation method to use the characteristic diagram area and the non-significant area intensity ratio significantly as the weight coefficient of the figure to form the finalized enhanced saliency map as the equation 5.

$$
S=\left[1 /\left(\sum_{i=0}^{3} w w_{i}\right)\right] \sum_{i=0}^{3} w w_{i} \cdot N\left(S_{i}\right)
$$


The Novel Change Detection Algorithm. Main process image change detection techniques are as the follows: image pre-processing, differences image acquisition and the image analysis. The image pre-processing mainly focus on image radiation correction and geometry correction, as far as possible, inhibition of the pseudo change. Difference image obtained mainly through pixels in different period of image contrast to get a picture after comparing the differences of images. As the novel perspective, we introduce the saliency detection procedure into the difference image generating step to enhance the efficiency. Difference method of image analysis is mainly to have differences in image segmentation, in this article, we form the final change detected image based on the difference image segmentation of fuzzy clustering with joint local information [4-5].

$$
\begin{aligned}
& J(U, V)=\sum_{i=1}^{c} \sum_{k=1}^{n}\left(u_{i k}\right)^{m}\left(d_{i k}\right)^{2} \quad k=1,2, \cdots n ; i=1,2, \cdots c \\
& u_{i k}=\left[\left(1 / d_{i k}^{2}\right)^{1 /(m-1)}\right] /\left[\sum_{j=1}^{c}\left(1 / d_{i k}^{2}\right)^{1 /(m-1)}\right] \\
& v_{i}=\left[\sum_{k=0}^{n} u_{i k}^{m} \cdot x_{k}\right] /\left[\sum_{k=0}^{n} u_{i k}^{m}\right]
\end{aligned}
$$

As demonstrated in the formulas 6 8, classical fuzzy clustering algorithm is analyzed. However, on this basis, the clustering of all the operations is based on the clustering center point to the distance change without considering that the point neighborhood pixels have effects on that point. This creates clustering algorithm very sensitive on isolated point and easily affected by noise. An important feature is the neighboring pixels of the image are mostly similar characteristic values and they belong to the same class of probability is higher. Therefore, neighborhood information will help improve quality of the clustering accuracy. We will adopt the weighted neighborhood pixels value changed from simple homogeneous weight to weight based on Euclidean distance space as made of the influence degree of the center pixel neighborhood pixels as the neighborhood pixels and the center pixel space changes of the Euclidean distance rather than simply taking the uniform weights. In the improved algorithm, the neighborhood pixels to the center pixel is set to the influence of different, for each pixel point in the neighborhood, the closer to the center pixel and if its position is greater the impact of it on the center pixel and the changing rule reflected in the neighborhood pixels on the weighted value.

$$
w_{l}=1 /\left(1+D_{k l}\right)
$$

As shown above, we define the weight and the $D_{k l}$ represents the space Euclidean distance between the center pixel and neighborhood pixels. Correspondingly, the other parameters are revised as below.

$$
\begin{aligned}
& \left(d_{i k}^{N_{k}}\right)^{2}=\sum_{l \in N_{k}} \frac{1}{1+D_{k l}}\left\|x_{l}-c_{i}\right\|^{2}+\left\|x_{k}-c_{i}\right\|^{2} \\
& u_{i k}^{\prime}=1 /\left[\sum_{j=1}^{c}\left(\frac{\sum_{l \in N_{k}} \frac{1}{1+D_{k l}}\left\|x_{l}-c_{i}\right\|^{2}+\left\|x_{k}-c_{i}\right\|^{2}}{\sum_{l \in N_{k}} \frac{1}{1+D_{k l}}\left\|x_{l}-c_{j}\right\|^{2}+\left\|x_{k}-c_{j}\right\|^{2}}\right)^{1 /(m-1)}\right]
\end{aligned}
$$

By using the algorithm to deal with the noisy image, when the noise is pixel neighborhood center, as it is affected by neighborhood around a lot of normal pixel, by automatically adjusting membership degree we can better avoid the misclassification. Differences at the same time, the use of pixel space position information could assist retaining more of the noise in the image details.

\section{Experiment and Simulation}

In order to assess the effectiveness and efficiency of our proposed saliency guided change detection algorithm, three main experiments are taken into consideration: (1) the effectiveness of the proposed local information assisted saliency detection methodology; (2) the comparison analysis on overall performance of the algorithms from the perspectives of false pixel, missing pixel and the Kappa index proposed by the literatures; (3) the time-consuming property of the different methods. 
From the figure one, the saliency regions are acceptable for further processing, the information of the original images are effectively extracted. From the figure two, we show the test images, ground truth references and the difference results. In the table one, we numerically simulate the algorithms with the corresponding statistical data. From the result, we could conclude that our algorithm outperforms compared with the other methodologies theoretically and numerically.
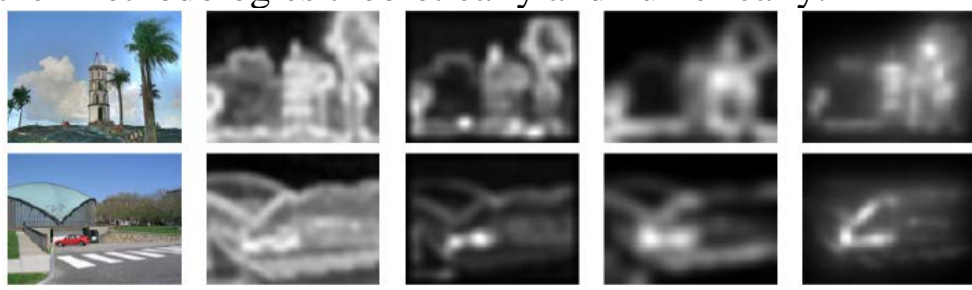

Fig. 1The Demonstration of the Visual Saliency Detection Methods
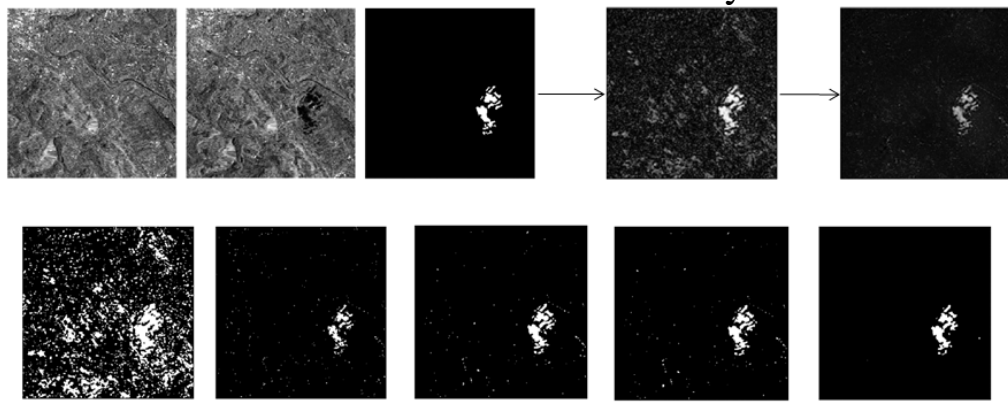

Fig. 2The Simulation Results on the Proposed Algorithm

Table 1The Numerical Comparison on the Different Change Detection Algorithms

\begin{tabular}{|c|c|c|c|c|c|}
\hline & Gaussian & NN & MTEP & FCM & Proposed \\
\hline False(\%) & 51.3 & 25.7 & 21.6 & 16.32 & 5.11 \\
\hline Miss(\%) & 26.5 & 16.8 & 14.3 & 9.37 & 3.95 \\
\hline Total(\%) & 43.9 & 19.3 & 17.3 & 10.52 & 4.37 \\
\hline Kappa Index & 0.121 & 0.466 & 0.512 & 0.637 & 0.852 \\
\hline Time(s) & 6.79 & 5.21 & 4.95 & 4.02 & 2.35 \\
\hline
\end{tabular}

\section{Summary}

In this paper, we have presented a novel visual saliency guided high resolution remote sensing image change detection algorithm which holds significant difference from classic methodologies. Firstly, for enhancing the efficiency, we optimize the saliency map extracting method with local and the global information to undertake the task of finalizing most attractive regions. Later, we adopt neighborhood information to modify the traditional fuzzy clustering algorithm and propose the novel noise robust clustering method to help extract the real changed regions (RCR). The experiment reflects that our novel saliency guided strategy can integrate advantages of clustering method and the saliency features which enhance the efficiency as well as the correctness.

\section{References}

[1] Tewkesbury, Andrew P., et al. "A critical synthesis of remotely sensed optical image change detection techniques." Remote Sensing of Environment 160 (2015): 1-14.

[2] Zheng, Yaoguo, et al. "Using Combined Difference Image and-Means Clustering for SAR Image Change Detection." Geoscience and Remote Sensing Letters, IEEE 11.3 (2014): 691-695.

[3] Bi, Chujian, Haoxiang Wang, and Rui Bao. "SAR image change detection using regularized dictionary learning and fuzzy clustering." Cloud Computing and Intelligence Systems (CCIS), 2014 IEEE 3rd International Conference on. IEEE, 2014. 
[4] Andre, Daniel, et al. "Very high resolution Coherent Change Detection." Radar Conference (RadarCon), 2015 IEEE. IEEE, 2015.

[5] Zhang, Han, et al. "Fast SAR Image Change Detection Using Bayesian Approach Based Difference Image and Modified Statistical Region Merging." The Scientific World Journal (2014). 